Résumés des conférences et travaux

Les représentations de l'espace en Occident de l'Antiquité tardive au XVI ${ }^{e}$ siècle

\title{
Cartographies médiévale et moderne et échanges interculturels
}

\section{Felicitas Schmieder}

\section{OpenEdition Journals}

Édition électronique

URL : https://journals.openedition.org/ashp/691

DOI : 10.4000/ashp.691

ISSN : 1969-6310

Éditeur

Publications de l'École Pratique des Hautes Études

Édition imprimée

Date de publication : 1 octobre 2009

Pagination : 148-150

ISSN : 0766-0677

Référence électronique

Felicitas Schmieder, "Cartographies médiévale et moderne et échanges interculturels », Annuaire de I'École pratique des hautes études (EPHE), Section des sciences historiques et philologiques [En ligne], 140 | 2009, mis en ligne le 15 octobre 2009, consulté le 06 juillet 2021. URL : http:// journals.openedition.org/ashp/691 ; DOI : https://doi.org/10.4000/ashp.691 


\title{
CARTOGR APHIES MÉDIÉVALE ET MODERNE ET ÉCHANGES INTERCULTURELS
}

\author{
Conférences de $\mathrm{M}^{\mathrm{me}}$ Felicitas SCHMIEDER, \\ professeur à la FernUniversität Hagen (Alemagne), \\ directeur d'études invité
}

\section{Représentation du temps dans l'espace}

Les mappemondes médiévales ne sont pas des représentations « correctes » de la terre au sens moderne. Elles montrent les trois parties de la terre en relation l'une à l'autre, mais on ne peut y reconnaitre ni mesures précises ni formes géographiques familières.

Spécialement dans le Moyen Âge tardif (époque d'où provient le plus grand nombre de mappemondes), la raison de leur production est toujours difficile à établir. Elles ne servaient pas au voyage, mais pourtant on tint, au cours des découvertes en Asie et ailleurs, à les adapter et les moderniser. Pour cette raison, des mappemondes reproduisent de façon exacte les côtes de l'Europe et de la Méditérranée et de plus en plus aussi le littoral africain, mais le reste du monde garde une représentation distordue selon la tradition plus ancienne.

Pour quelle raison ces images étaient-elles capables de se moderniser sans sacrificier les formes et les contenus antérieurs? On a décrit les mappemondes comme des sortes de chroniques universelles en image, parce qu'elles représentent non seulement l'espace mais aussi le temps depuis la Genèse jusqu'à la fin du monde. Pour le temps du passé et même probablement du présent, une mappemonde en tout traditionelle suffisait, mais il n'en allait pas de même pour les temps à venir. Dieu ayant communiqué certains signes inscrits dans le monde qui pouvaient être lus et interprétés en donnant un sens au passé, au présent, et au futur de l'humanité, les chroniques universelles étaient parfois accompagnées de mappemondes qui manifestaient ces signes de façon tangible en en faisant des porteurs de sens eschatologique. Les mappemondes ainsi intégrées dans des chroniques universelles, les deux instruments se renforçaient mutuellement, et la modernisation trouvait sa raison d'être et sa justification dans cette orientation eschatologique, la correspondance des temps devant s'étendre au présent et à l'espace dans lequel il se déployait.

\section{Cartographie religieuse}

D'autres éléments d'origine religieuse trouvent leur expression dans les mappemondes. Un des événements centraux à venir est le fait de l'évangélisation complète du monde, qui se trouve représenté très tôt sur les cartes du Commentaire de l'Apocalypse de Beatus de Liébana montrant les tombes des douze apôtres répandus dans le monde entier. 
Mais ce n'est pas seulement le christianisme qui était figuré. Les auteurs des mappemondes se préoccupaient aussi de faire place aux autres religions, surtout en rapport avec leur expansion dans l'ensemble de l'espace géographique. À la fin du Moyen Âge en particulier, les contacts et les rapports de concurrence des différentes religions purent être représentés. En conséquence de la connaissance élargie du monde du fait des croisades et des voyages en Asie, un certain pessimisme pour la situation de la Chrétienté et pour les lourdes pertes qu'elle avait subies s'exprima, par comparaison avec la situation passée beaucoup plus favorable. En même temps, l'optimisme était susceptible de s'alimenter du grand nombre de foyers chrétiens répandus sur l'ensemble de la terre habitée.

Innocent IV († 1254) avait déjà prétendu à la souveraineté papale sur l'ensemble du monde, qu'il soit chrétien ou non. On peut considérer certaines mappemondes du Moyen Âge tardif comme figurant l'espace où devait se réaliser la réalisation de cette tâche confiée au pape et aux princes chrétiens. L'exemple central pour cela est la mappemonde dite de Velletri gravée sur une table de bronze d'environ $65 \mathrm{~cm}$ de diamètre. S'y associent les souvenirs du passé biblique et l'espérance du futur, mais surtout elle montre un catalogue des religions du monde présent, avec notamment des notions sur l'islam et sur les ennemis de la foi aux frontières de l'Europe.

\section{Cartographie et échanges interculturels}

La mappemonde transmise au Japon et en Corée, intitulée en japonais Kon'itsu kyōri rehidai kokuto no zu, a permis de réfléchir aux influences mutuelles des deux fins du monde connu en Moyen Âge - Europe et Asie orientale - sous l'effet des échanges culturels dans l'Empire mongol. À partir du milieu du XIII ${ }^{\mathrm{e}}$ siècle, les Mongols ont occupé une grande partie de l'Asie, tandis que l'Ouest et l'Extrême-Orient, bien qu'inoccupés par eux, ne restèrent pas intouchés par leur culture. Les Mongols se souciaient de rassembler les meilleurs spécialistes qu'ils rencontraient dans les territoires qu'ils contrôlaient et de les faire travailler dans l'intérêt de leur domination. Les conséquences se répandirent à la périphérie de l'empire et au-delà de ses limites, phénomène que l'on a appelé «the Mongol experience », dont de nombreux peuples ressentirent les effets..

Parmi d'autres (comme les dictionnaires de langues, tel le codex Cumanicus et l'historiographie universelle, telles les chroniques universelles du franciscain Paulin de Venise dans la première moitié du XIV ${ }^{\mathrm{e}} \mathrm{s}$. et de Rashid ad-Din, vers 1300 à la cour de l'Ilkhan mongol de Perse), il est possible de montrer la réalité de ces processus d'échanges mettant en relation l'Europe latine, le monde arabo-musulman et l'empire mongol. La carte japonaise ou coréenne, quant à elle, reproduit des modes de représentation arabe, l'exemple le plus probant étant constitué par les sources du Nil qui se retrouvent sur la carte d'Idrisi (vers 1150 à la cour du roi Roger II de Sicile).

Au début de l'époque moderne, un phénomène similaire d'un échange direct entre Europe et Extrême-Orient est illustré par deux cartes du monde d'origine japonaise, l'une dessinée par un jésuite, l'autre par un boudhiste. La première transcrit le savoir européen contemporain, mais place l'océan Pacifique au centre de façon à correspon- 
dre aux conceptions asiatiques ; l'autre dessine l'Europe au bord de la terre tout en plaçant le mont Meru au centre.

\section{Le voyage virtuel}

À la fin du Moyen Âge, l'image du monde pouvait être aussi manifestée par des descriptions de voyage « global » émanant aussi bien de voyageurs ayant vu au moins une part de ce qu'ils décrivent, que d'auteurs rassemblant dans leur cabinet de travail des informations tirées des récits de voyage ou des mappemondes. La différence entre les deux procédés n'est pas toujours facile à établir (l'exemple le plus connu est celui de Jean de Mandeville).

Tous ces auteurs réels ou fictifs devaient résoudre une même difficulté d'ordre intellectuel : situer les régions dans leur contexte en décrivant leurs rapports, leurs points et lignes de contact et leurs positions réciproques et en remplaçant par des notions plus précises le flou qui entourait les régions les plus lointaines.

Des poètes de la fin du Moyen Âge, des auteurs de traités politiques et des géographes procurent des descriptions du monde dont le caractère virtuel est plus ou moins affirmé. On a comparé de ce point de vue des passages du « Niederrheinischer Orientbericht » $(\mathrm{ca}$ 1350) et de la description de Terre sainte de Francesco Suriano ( ca 1500), du Chemin de longue estude de Christine de Pisan (début $\mathrm{XV}^{\mathrm{e}}$ siècle) et du Songe du vieil pelerin de Philippe de Mézières (1389) en considérant leurs idées respectives de la cohérence géographique et les moyens qu'ils ont mis en œuvre pour la réaliser et produire chez leurs lecteurs une image ordonnée du monde. 